\title{
Design of Light Source System and Optical System for a Static Star Simulator
}

\author{
Yunhui Hao \\ ChangChun University of Science and Technology, ChangChun, China \\ haoyunhui3@sina.com
}

\begin{abstract}
This paper introduced a scheme of using cold cathode planar backlight, and presented the power supply circuit for light source. According to the analysis, parameters of static star simulator were determined, and then a large field, small distortion and small field curvature collimating objective lens was designed by using transmission optical system. The imaging analysis results show that the design can satisfy the request.
\end{abstract}

Keywords: star simulator; backlight; power supply circuit; collimating objective lens

\section{Introduction}

Star sensors have the highest accuracy for obtaining the real-time attitude of aircrafts, the application prospect is broad, and so the requirements of accuracy and reliability are higher and higher [1]. Star simulators are the test and calibration equipment for star sensors on the ground, they simulate stars as observation targets of star sensors, and so star sensors can be detected in the laboratory to avoid the influence of natural conditions.

Static star simulators usually simulate size, star magnitude, spectral property and color temperature of stars [2]. This paper designed a miniature static star simulator which is lighter than before, and its field of view is larger.

\section{Principle of Static Star Simulators}

The lights whose brightness is uniform irradiate on the star plate through the filter, the star plate is located in the focal plane of the optical system. There are some holes on the star plate to simulate stars. The lights through the holes and emitted by the optical system to simulate static star map at the entrance pupil of star sensors as the observation target. The principle diagram is shown in Figure 1.

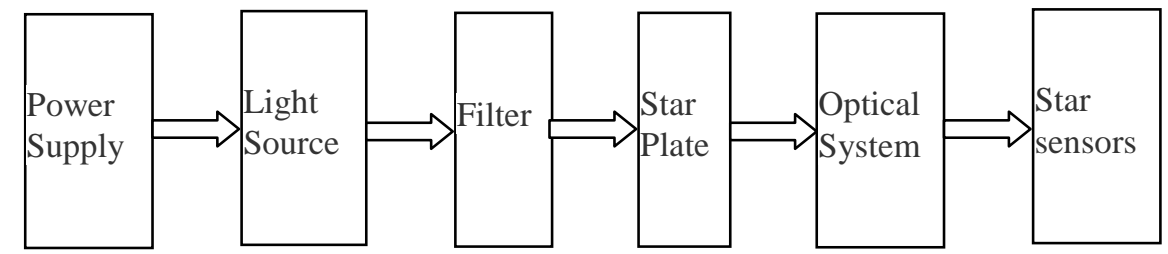

Figure 1. The Principle Diagram of Static Star Simulator

\section{Light Source System of the Static Star Simulator}

\subsection{Light Source System Features}

Radiation characteristics of stars include brightness and spectrum of stars, and these characteristics can be simulated by light source. 
Brightness characteristics of stars mainly have three points: (1) stars are weak light sources; (2) the number of dim stars is much more than the number of bright stars; (3) the brightness of stars has great difference in the sensitive range of a star sensor. Simulators simulate brightness and spectrum of stars to test the detection sensitivity and photometric response of star sensors.

The detection sensitivity determines the number of stars which can be captured in the field of view of a star sensor [3]. Photometric response characteristic is the relationship between response output of a star sensor and incident intensity, some software algorithm of star sensors depend on the determination results of photometric response characteristic.

Spectral characteristics of stars mainly have three points: (1) the spectral range of a star is very wide; (2) most of spectrums of stars are close to the radiation spectrum of the blackbody; (3) spectrums of different types of stars have great difference. Simulators simulate spectrum of stars to test the spectral response characterization of star sensors [4].

Star sensors only having response to the incident light within a certain wavelength range due to the limitation of optical system and optoelectronic components, star simulators only need to simulate spectrum of stars in this range. The spectral range of this star simulator is $0.5 \mu \mathrm{m}$ to $0.8 \mu \mathrm{m}$, center wavelength is $0.65 \mu \mathrm{m}$, light source stability is better than 0.03 per hour.

According to the analyses above, the light source should have adjustable brightness, high stability, and the luminescence spectra should satisfy the requirement of the star sensor.

\subsection{Design of Light Source System}

In order to simplify the structure of the static star simulator, and ensure uniform brightness, the star simulator selected cold cathode planar backlight as light source. Adjusting the supply voltage of backlight can change its luminous intensity [5]. The backlight basically has no fever at work, so it avoids the luminescent spectrum offset when the power supply is stable. Its main performance indexes are as follows: (1) color: white light; (2) brightness: $300 \mathrm{~cd} / \mathrm{m}^{2} \sim 3000 \mathrm{~cd} / \mathrm{m}^{2}$; (3) luminance uniformity: $\geq 85 \%$; (4) working voltage: $3 \mathrm{~V}$; (5) working current: $30 \mathrm{~mA}$; (6) service life: 50000 hours $\sim 100000$ hours; (7) storage temperature: $243.15 \mathrm{~K} \sim+353.15 \mathrm{~K}$; (8) working temperature: $253.15 \mathrm{~K} \sim$ $+343.15 \mathrm{~K}$..

The static star simulator used $24 \mathrm{~V}$ directed current power for the backlight, and equipped a button battery in the battery compartment as standby power supply in case of no $24 \mathrm{~V}$ directed current power at the scene. The output voltage of power-supply modes both were $3 \mathrm{~V}$ and the output current could be changed through the precision potentiometer, so the supply voltage of the backlight would be adjusted to change its brightness.

The exterior power supply was $24 \mathrm{~V}$ directed current power, but the working voltage of the backlight was $3 \mathrm{~V}$, so there must be a circuit to achieve the change from $24 \mathrm{~V}$ to $3 \mathrm{~V}$. In this system, LM317T which is a three-terminal voltage regulator was chosen, and the circuit of the exterior power supply is shown in Figure 2.

LM317T is an adjustable integrated voltage regulator, its output voltage is continuously adjustable from $1.2 \mathrm{~V}$ to $37 \mathrm{~V}$, and the output can be set by external resistors. The output voltage can be calculated by (1).

$$
V_{\text {out }}=1.25\left(1+\frac{R_{1}}{R_{2}}\right)
$$




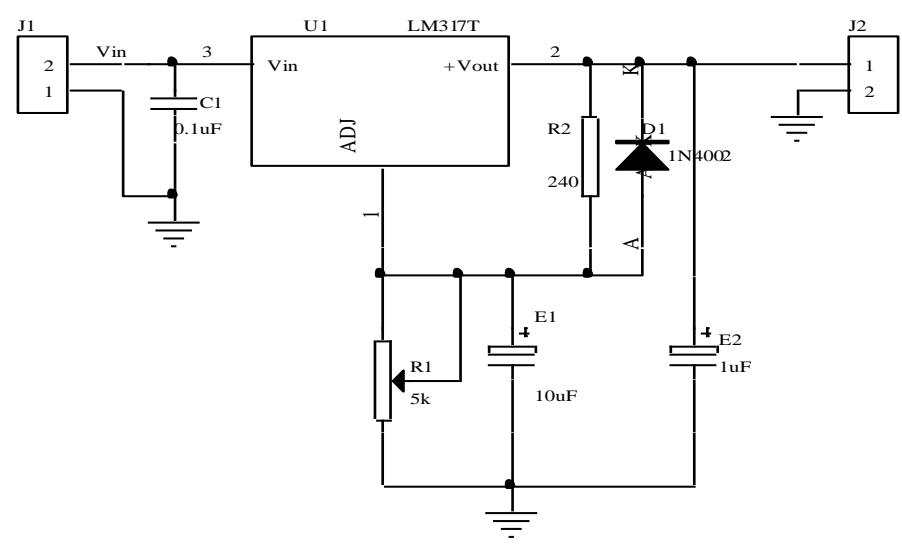

Figure 2. The Exterior Power Supply Circuit

In the electrical circuit, the external capacitor $\mathrm{C}_{1}$ at the input was used for power supply filtering, and it was beneficial to improve the ability of ripple suppression. The external capacitor $\mathrm{E}_{2}$ at the output can eliminate the oscillation and ensure the circuit work stably. $\mathrm{E}_{1}$ could improve the ripple suppression characteristics of voltage stabilizer LM317T. When $\mathrm{E}_{1}$ was $10 \mu \mathrm{F}$, the ripple rejection ratio of DC power supply which was outputted by LM317T would be $80 \mathrm{~dB}$. But $\mathrm{E}_{1}$ could not be too big otherwise the integrated stable voltage was easy to produce self-excited oscillation ${ }^{[6]}$. $\mathrm{D}_{1}$ between $\mathrm{V}_{\text {out }}$ and ADJ provided a discharge path for $\mathrm{E}_{1}$ to prevent LM317T from being damaged by discharge current when output was a short circuit.

The system requires that the star simulator could simulate the spectral range of stars is $0.5 \mu \mathrm{m}$ to $0.8 \mu \mathrm{m}$, center wavelength is $0.65 \mu \mathrm{m}$, so we used the optical filter which was made by vacuum coating to modify the luminescence spectra of backlight. The optical filter is a band-pass filter, and its transmittance of passband is more than 95\%. The transmittance graph of the filter is shown in Figure 3.

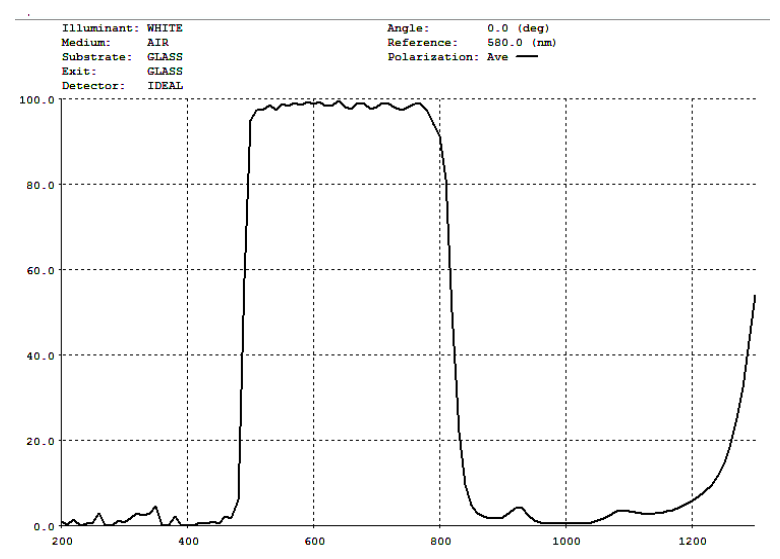

Figure 3. The Transmittance Graph of the Filter

\section{Optical System of Static Star Simulator}

\subsection{Optical System Features}

Stars have minimal field angles because they are very far away from the earth, they can be seen as point light sources [7], starlight arrived the earth can be seen as parallel light. So the optical system is a collimating objective which exits parallel light to simulate stars at infinity, and then the lights are received by the star sensor. 
In order to ensure the process of transferring lights from the star simulator to the star sensor without loss of light energy, the optical systems of both the two equipment should have the same optical axis, and the exit pupil of the collimating objective should coincide with the entrance pupil of the star sensor.

Because the field of view of the star map should be $20^{\circ} \times 20^{\circ}$, so the collimating objective system must have large field of view. It also has small distortion, small field curvature and apochromatic imaging quality in order to ensure all star points in the field of the simulated star map have accurate location and constant magnitude.

The optical system parameters of the star simulator are determined by the unified consideration of the optical system parameters of the star sensor and the star simulator [8]. The exit pupil of the collimating objective should be outside the optical system because that the exit pupil of the collimating objective coincides with the entrance pupil of the star sensor, the exit pupil distance is determined by the position of the star sensor and the distance between the star simulator and the star sensor. The exit pupil of the collimating objective is the same size as the entrance pupil of the star sensor, and its field of view is slightly larger than the field of the star sensor.

Due to the requirement of the large field of view, the collimating objective was designed by the transmission optical system, and corrected aberration by $\mathrm{F}$ line, $\mathrm{C}$ line and $\mathrm{r}$ line. The main parameters of the collimating objective are shown in table 1.

Table 1. The Main Parameters of the Collimating Objective

\begin{tabular}{|c|c|}
\hline Effective field of view & $28.3^{\circ}$ \\
\hline Focal length & $60 \pm 1 \mathrm{~mm}$ \\
\hline Spectral range & $0.5 \mu \mathrm{m} \sim 0.8 \mu \mathrm{m}$ \\
\hline Center wavelength & $0.65 \mu \mathrm{m}$ \\
\hline Effective aperture & $\Phi 25 \mathrm{~mm}$ \\
\hline Exit pupil distance & $20 \mathrm{~mm}$ \\
\hline Distortion & $<0.1 \%$ \\
\hline
\end{tabular}

\subsection{Structure of Collimating Objective}

The focal length of this collimating objective is $59.97 \mathrm{~mm}$, rear cut-off distance is $13.96 \mathrm{~mm}$, total length of the optical system is $110.56 \mathrm{~mm}$, and image height is $30.26 \mathrm{~mm}$. The structure of the optical system for collimating objective is shown in Figure 4.

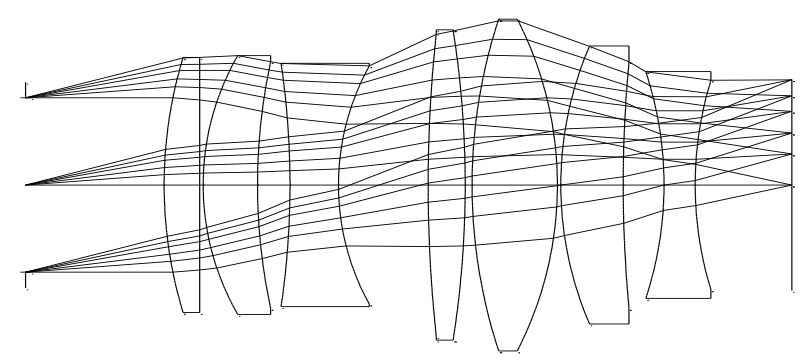

Figure 4. The Structure of the Optical System for Collimating Objective

\subsection{Image Quality Evaluation}

The image quality of the optical system could reflect the situation of the system aberration correction, and then reflect whether the optical system meets the requirements. The system of collimating objective was designed by optical design software ZEMAX, and 
axial spherical aberration, vertical axis aberration, distortion, and spot diagram were analyzed through three different wavelengths of lights $0.486 \mu \mathrm{m}, 0.656 \mu \mathrm{m}$, and $0.707 \mu \mathrm{m}$.

Figure 5 longitudinal aberration shows that the $\mathrm{C}$ light spherical aberration is almost zero from 0 degree visual field to a large visual field, and it is the biggest in the full field of view. The system has corrected spherical aberration, and corrected color difference in the 0.81 visual field to $\mathrm{F}$ light and $\mathrm{r}$ light, but the optical system still has secondary spectrum. The secondary spectrum is less, and it could satisfy the use requirement.

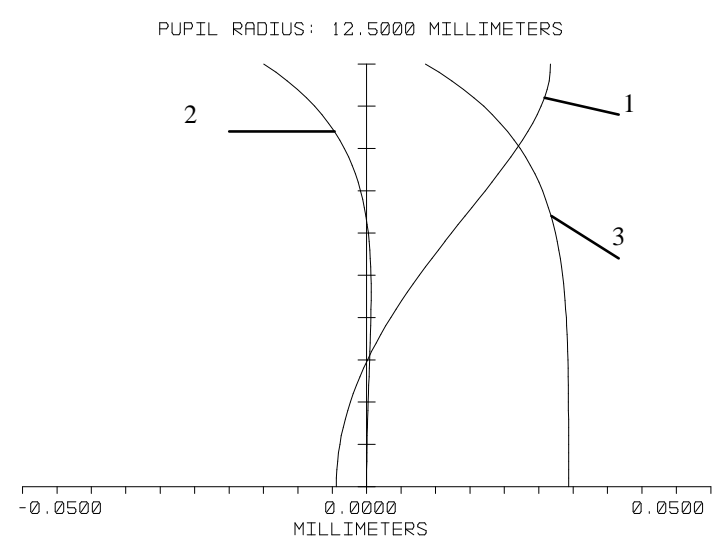

Figure 5. Longitudinal Aberration

1 - F light $(0.486 \mu \mathrm{m}), 2$ - C light $(0.656 \mu \mathrm{m}), 3$ - $\mathrm{r}$ light $(0.707 \mu \mathrm{m})$

Distortion reflects aberration of the main light, and it does not affect the definition of imaging, but makes the image become deformed. The field curvature and distortion are shown in Figure 6, and the distortion values of $\mathrm{C}$ light are shown in Table 2. It can be seen that the field curvature, astigmatism and distortion of this optical system of collimating objective are all small.
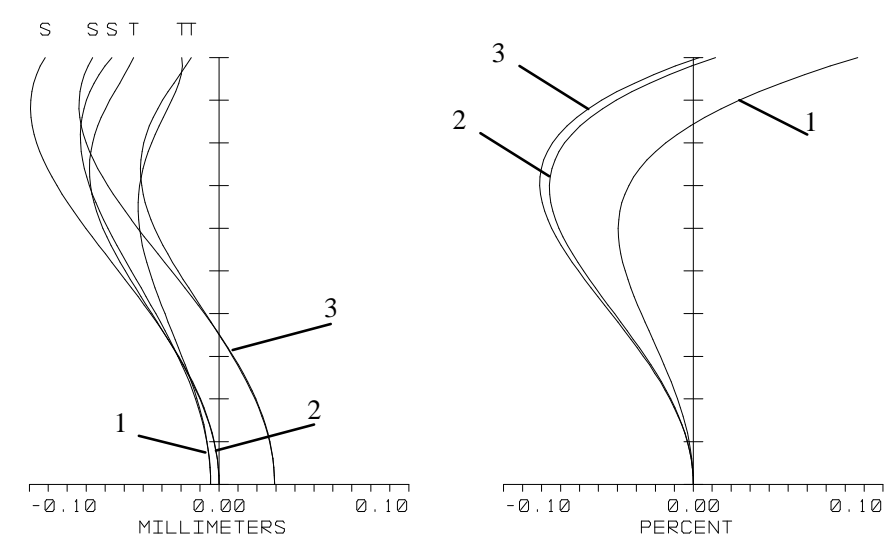

Figure 6. Field Curvature and Distortion

1 - F light $(0.486 \mu \mathrm{m}), 2$ - C light $(0.656 \mu \mathrm{m}), 3$ - r light $(0.707 \mu \mathrm{m})$ 
Table 2. Distortion Value of the Collimating Objective

\begin{tabular}{|c|c|c|c|c|}
\hline Number & $\begin{array}{c}\text { Relative image } \\
\text { height }\end{array}$ & $\begin{array}{c}\text { Half image } \\
\text { height }\end{array}$ & Distortion & $\begin{array}{c}\text { Relative } \\
\text { distortion(\%) }\end{array}$ \\
\hline $\mathbf{1}$ & 1 & 15.118 & 0.00177 & 0.012 \\
\hline $\mathbf{2}$ & 0.85 & 12.773 & -0.00724 & -0.057 \\
\hline $\mathbf{3}$ & 0.707 & 10.581 & -0.00801 & -0.076 \\
\hline $\mathbf{4}$ & 0.50 & 7.443 & -0.00431 & -0.058 \\
\hline $\mathbf{5}$ & 0.30 & 4.451 & -0.00113 & -0.025 \\
\hline
\end{tabular}

The values show that in 0.707 field of view, the distortion reaches the maximum of $0.076 \%$, less than $0.1 \%$, so the distortion satisfy the requirement of the system.

The transverse ray fan plot is shown in Figure 7. It chooses six fields of view: 0 degree, 4.25 degree, 7.08 degree, 10 degree, 12.03 degree and 14.15 degree. It shows that coma increases with field of view, and the maximum value is about $0.018 \mathrm{~mm}$ in the full field of view, the system has corrected the coma.
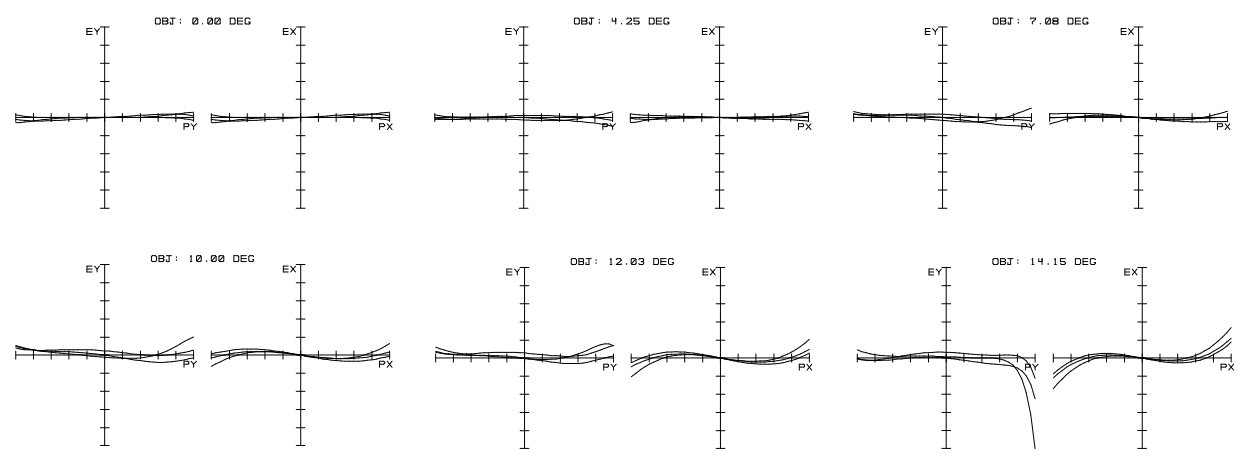

Figure 7. Transverse Ray Fan Plot (maximum scale: $\pm 100 \mu \mathrm{m}$ )

The spot diagram is shown in Figure 8. RMS radius and geometry radius of every field of view are shown at the bottom of the diagram. RMS radius in the spot diagram is the root mean square of the distance of light and reference point, it can give a rough idea of light diffusion because it depends on each light. Geometry radius is the maximum distance between light and reference point, it only gives the information of the light which is farthest from reference point. It can be seen that the dispersion spot diameters in all the fields of view are small.
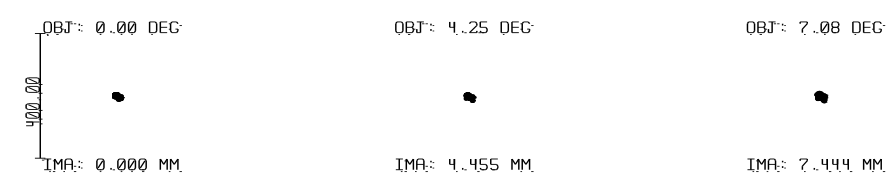

$\mathrm{OBT}=10.0 \square \mathrm{DEG}$

$\mathrm{OBJ}:=1.2 .03 \quad \mathrm{DEC}$

DBST: 1.4 .1 .5 DEG

IMP: $: 10.566 \mathrm{MM}$

IMP:: $1.2 .772 . \mathrm{MM}$.

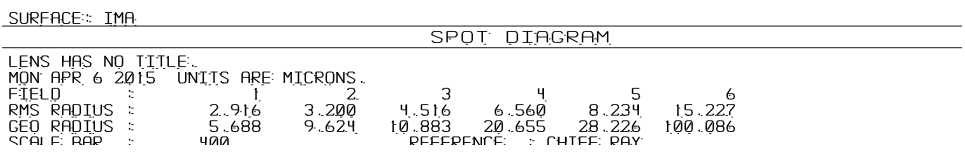

Figure 8. Spot Diagram 
Because star sensors determine attitude by looking the energy center of star images as ideal reference, so the position deviation of energy center and the main light will affect the precision of angular distance between stars. The angle error can be calculated by (2), $d$ is the position deviation of energy center and the main light, $f^{\prime}$ is the focal length of the optical system. Angle errors because of the position deviation of energy center and the main light are shown in Table 3.

$$
\theta=\frac{d}{f^{\prime}} \times 206265
$$

Table 3. Angle Errors

\begin{tabular}{|c|c|c|}
\hline $\begin{array}{c}\text { Field of view } \\
\text { (Normalization) }\end{array}$ & $\begin{array}{c}\text { Position deviation of } \\
\text { energy center and the } \\
\text { main light }(\boldsymbol{\mu m})\end{array}$ & $\begin{array}{c}\text { Angle errors } \\
(\text { ") }\end{array}$ \\
\hline $\mathbf{0}$ & 0.0 & 0.0 \\
\hline $\mathbf{0 . 3}$ & 0.81488 & 2.80 \\
\hline $\mathbf{0 . 5}$ & 0.02826 & 0.01 \\
\hline $\mathbf{0 . 7 0 7}$ & 2.3808 & 8.19 \\
\hline $\mathbf{0 . 8 5}$ & 3.8246 & 13.15 \\
\hline $\mathbf{1}$ & 1.2272 & 4.22 \\
\hline
\end{tabular}

\section{Conclusion}

This paper has designed a light source system which can adjust the brightness to simulate the change of star brightness through adjusting the supply voltage of the light source, and designed an optical system of collimating objective using seven separated collimating lens. The field of view of the collimating objective is 28.3 degree, focal length is $59.97 \mathrm{~mm}$, total length is $110.56 \mathrm{~mm}$, and distortion is $0.076 \%$. The system meets the design requirements.

\section{Acknowledgments}

The author thanks the key laboratory of photoelectric measurement and control and optical information transmission technology (Changchun University of Science and Technology) of Ministry of Education for support provided. Thanks Prof. Guoyu Zhang for his helpful guidances.

\section{References}

[1] C. C. Liebe, “Aerospace and Electronics Systems Magazine”, IEEE, (1995), pp. 10-16.

[2] W. Zhang, X. Wang, Y. Ma and H. Duan, "Opto-Electronic Engineering”, vol. 25, (1998), pp. 74-78.

[3] U .Schmidt, "European Space Agency", (Special Publication) ESA SP, vol. 554, no. 6, (2004), pp. 355358.

[4] F. Jianying, Y. Wang and G. Guo, "Journal of harbin University of Science and Technology", vol. 14, no.6, (2009), pp. 1-4.

[5] Z. Yu, T. Sun, L. Li and S. Zhang, "Microcomputer Information", vol. 23, no.2, vol. 8, (2007), pp. 300302.

[6] Q. Liu, Y. Sun and S. Cai, Journal of Hebei University (Natural Science Edition), vol. 21, no. 2, (2001), pp. 176-178.

[7] Z. Wang and Y. Zhang, "Journal of System Simulation”, vol. 18, no. 5, (2006), pp. 1195-1198.

[8] Z. Chen, Editor, "The emulation test system of star sensor based on real-time star field simulator", Proceedings of System Simulation Technology and Application, (1995); Ningbo, China. 
International Journal of Smart Home

Vol. 9, No. 11, (2015) 